\title{
Correlações entre os atributos climáticos e a morbidade hospitalar por doenças isquêmicas do coração na cidade de João Pessoa-PB
}

\author{
Correlations between the climatic attributes and hospital morbidity from ischemic heart \\ disease in the city of João Pessoa-PB
}

CAVALCANTE, G. P.; MOURA, M. O.

cavalcantegp.geo@gmail.com

\begin{abstract}
Resumo
O presente artigo tem como objetivo verificar o grau de associação entre as médias mensais dos atributos climáticos (insolação, temperatura máxima do ar, dias com precipitação e umidade relativa do ar) e as taxas de internações hospitalares por Doenças Isquêmicas do Coração entre pacientes idosos residentes na cidade de João Pessoa no período entre 2000 a 2015. Utilizou-se o Coeficiente de Correlação de Pearson para obtenção dos coeficientes de correlação entre as variáveis, seguido da aplicação do teste $\mathrm{t}$ de Student para a verificação de significância estatística dos coeficientes. Constatou-se que as médias mensais dos atributos climáticos não produziram um impulso de natureza ambiental no aumento ou na diminuição da morbidade hospitalar por Doenças Isquêmicas do Coração entre os pacientes idosos.
\end{abstract}

Palavras-chave: Clima e saúde, Doenças

Cardiovasculares, Idosos.

\begin{abstract}
This article aims to verify the degree of association between the monthly averages of climatic attributes (insolation, maximum air temperature, days with precipitation and relative humidity) and hospitalizations occurred for Ischemic Heart Diseases among elderly patients in the city of João Pessoa for the period 2000 2015. We used the Pearson correlation coefficient for obtaining the correlation coefficients between the variables, followed by the application of the Student $t$ test for verification of statistical significance of the coefficients. It was found that the monthly averages of climatic attributes did not produce an environmental boost in increased or decreased morbidity for Ischemic Heart Diseases among elderly patients.
\end{abstract}

Keywords: Climate and Health, Cardiovascular Diseases, Elderly.

\section{INTRODUÇÃO}

O estudo dos tipos de tempo e do clima e sua relação com estado de saúde e de doença das populações não é assunto novo nas produções científicas do país, visto que tal discussão surgiu no Brasil nas primeiras décadas do século $\mathrm{XX}$ de forma atrelada às primeiras discussões acerca do clima em sua essência integral ou puramente natural/determinista (SANT’ANNA NETO, 2015). Nas duas últimas décadas surgiu uma preocupação no mundo acadêmico acerca da relação entre as variáveis climáticas e a incidência de doenças crônicas, preocupação que seu deu devido às projeções das mudanças climáticas globais, mas também por conta das alterações do clima das cidades e dos hábitos contemporâneos da população. As cidades cresceram e o modo de vida da população mudou com o passar dos anos, com adoção de um estilo de vida sedentário e maus hábitos alimentares. Concomitante a isso, com a evidência das mudanças climáticas, aumentou a preocupação dos pesquisadores acerca dessas novas condições ambientais sobre o estado de saúde e de doença da população.

${ }^{1}$ Gabriel de Paiva Cavalcante, graduando do curso de Bacharelado em Geografia/CCEN/UFPB, membro Laboratório de Climatologia Geográfica (Climageo)/Departamento de Geociências, Universidade Federal da Paraíba, João Pessoa-PB, Brasil. 
A análise das relações entre as variáveis climáticas e a morbimortalidade por doenças crônicas, a exemplo das doenças cardiovasculares e respiratórias, em especial, nos espaços urbanos, tem sido pauta de investigação nos grandes centros de pesquisa do mundo (BESANCENOT, 2002; WHO, 2004; MCMICHAEL et al., 2008), uma vez que fatores como temperatura e umidade do ar afetam diretamente a população, causando sensação de desconforto térmico e agravando algumas doenças pré-existentes através de variações fisiológicas consideráveis (PEREIRA et. al., 2011).

As variações fisiológicas, entre outros fatores, podem ser anunciadas por períodos térmicos extremos e ocorrem mais facilmente em pessoas idosas. A fragilidade fisiológica dos idosos, seguida, frequentemente, de polipatologia, e a sua incapacidade progressiva de assegurar uma termólise eficaz é uma das razões que explica sua vulnerabilidade frente aos riscos climáticos térmicos de natureza extrema (BESANCENOT, 2002).

As Doenças Cardiovasculares - DCV, sobretudo, o grupo das Doenças Isquêmicas do Coração, grupo que engloba o Infarto Agudo do Miocárdio, tipo de patologia que de acordo com Besancenot (1986, 2001, 2002) é por excelência uma doença meteorossensível, são as doenças cardíacas que têm mais relação com as variáveis térmicas. Dessa forma os portadores de DCV podem apresentar um quadro de agravo (internação hospitalar e até mesmo de óbito) disparado por eventos térmicos extremos do ar.

A cidade de João Pessoa vem registrando uma tendência, que também é nacional, de aumento da proporção de idosos sobre a população geral, o que acarreta em uma demanda cada vez maior do poder público no que tange à disponibilidade de serviços de saúde mais isonômicos, especializados e com capacidade de atendimento. Em 2000, 8,14\% da população de João Pessoa era composta por idosos; tal proporção aumentou para $10,31 \%$ no ano de 2010 , ou seja, um aumento de $2,17 \%$ da população idosa, valor demográfico que significa, em números absolutos, um aumento de 25,9 mil idosos em um intervalo de 10 anos (IBGE, 2015). Fica evidente, desta forma, a necessidade iminente de adequação da rede hospitalar para o atendimento desse grupo populacional.

De acordo com os dados disponíveis no DATASUS (BRASIL, 2016), no intervalo entre os anos de 2000 e 2015, as doenças cardiovasculares (Classificadas pela 10 ${ }^{\mathrm{a}}$ Revisão (CID 10) da Classificação Internacional de Doenças como Doenças do Aparelho Circulatório: Capítulo IX, sob o código I00 - I99) representaram a quarta causa de morbidade hospitalar para a cidade de João Pessoa, com registro total de 58.842 internações; desse montante, 30.994 internações foram efetivados por idosos $(52,67 \%$ do total). Para o grupo de Doenças Isquêmicas, foram registradas 6.560 internações, sendo o Infarto Agudo do Miocárdio a quarta causa de internações entre os idosos (1.556 internações), e Outras Doenças Isquêmicas do Coração (5.004 internações), a terceira, também entre os idosos. 
Com base na conjuntura apresentada, definimos como objetivo central deste artigo: verificar correlações, em escala mensal, entre os atributos climáticos e a morbidade hospitalar por doenças isquêmicas do coração de pacientes idosos, residentes e atendidos em hospitais públicos e conveniados ao SUS na cidade de João Pessoa-PB no período entre 2000 a 2015.

\section{METODOLOGIA}

$\mathrm{Na}$ verificação das correlações entre as variáveis meteorológicas e a morbidade hospitalar por DCV duas abordagens são necessárias: a climática e a epidemiológica.

\section{1 - Dos atributos climáticos}

Realizou-se um estudo exploratório da série mensal dos atributos insolação, dias com precipitação, umidade relativa do ar e temperatura máxima do ar de 2000 a 2015. Como as alterações fisiológicas nos idosos são mais sensíveis através das temperaturas máximas do ar, visto que o conforto térmico é fator essencial para o bem-estar da pessoa idosa, tal atributo foi escolhido para a análise climatopatológica. Assim, para a identificação e a caracterização das temperaturas máximas do ar em seus registros mensais foram executadas as seguintes etapas: 1) Obtenção dos dados históricos no Banco de Dados Meteorológicos para Ensino e Pesquisa - BDMEP do Instituto Nacional de Meteorologia - INMET da Estação Meteorológica de João Pessoa; 2) Confecção de tabelas no ambiente do Microsoft Excel 2010 (Microsoft Corporation), no formato de planilhas eletrônicas, para a sistematização da base de dados e verificação de falhas da série, além da tabulação dos dados e 3) Cálculo da média mensal das temperaturas máximas do ar.

\section{2 - Da morbidade hospitalar}

Os dados de morbidade hospitalar foram obtidos através do Departamento de Informática do SUS (DATASUS). Foram coletados os registros de internações mensais, do período de 2000 a 2015, de pacientes residentes e atendidos em hospitais públicos e conveniados ao Sistema Único de Saúde - SUS na cidade de João Pessoa.

Neste trabalho, serão analisados os registros de internações de Doenças Isquêmicas do Coração em pessoas idosas. De acordo com a literatura, esse grupo de patologias cardíacas é um dos que mais têm relação com as variáveis climáticas (BESANCENOT, 2001, 2002; PAIXÃO; NOGUEIRA, 2003; MURARA; COELHO; AMORIM, 2010; PEREIRA et al., 2011; MOURA, 2013, 2015).

Após a obtenção dos dados hospitalares foram executadas duas etapas, a saber: 1) Processo de tabulação dos dados mensais de internações hospitalares por DCV, com filtro para os dois grupos de 
doenças isquêmicas do coração (Infarto Agudo do Miocárdio e Outras Doenças Isquêmicas do Coração, código I20 a I25) e 2) Cálculo da Taxa de Internação ou Taxa de Morbidade por 10 mil habitantes (Equação 1) para todos os meses do período de 2000 a 2015, conforme sugestão de Lima, Pordeus e Rouquayrol (2013):

Taxa de Morbidade $=\frac{\text { № de casos de uma doença }}{\text { populaģૈâ }} \times 10.000$

\section{3 - Dos recursos estatísticos para análise das correlações}

Os dados das variáveis climáticas, juntamente com os dados epidemiológicos, foram submetidos à análise por meio do Coeficiente de Correlação de Pearson (r), que identifica o grau de relação mútua entre duas variáveis. Com valores entre +1 e -1 , as correlações mais próximas desses valores são consideradas como Fortes, sendo que +1 é uma correlação proporcional perfeita, -1, uma inversamente proporcional perfeita e 0, correlação nula. (FIGUEIREDO FILHO; SILVA JÚNIOR, 2009 e GALVÃO et. al., 2015). No que diz respeito à avaliação da significância de tais correlações, será utilizado o teste $\mathrm{t}$ de Student. Neste teste, para que a correlação seja significante, o valor de $\mathrm{t}$ deve ultrapassar os valores críticos de significância. Assim, a hipótese de H0 é rejeitada e a correlação é estatisticamente significante (TRIOLA, 2008).

\section{RESULTADOS E DISCUSSÃO}

Foram abordados, em escala mensal, os atributos climáticos que consideramos possuir maior potencialidade de interferência na morbidade hospitalar por DCV entre idosos: total de horas de insolação, dias com precipitação, umidade relativa do ar e temperatura máxima. Este último atributo, segundo a literatura, é o que mais exige capacidade de resposta do organismo humano, causando desconforto térmico, especialmente nos idosos. A Prancha 1 mostra a distribuição mensal dos atributos climáticos durante o período de 2000 a 2015.

A distribuição das variáveis apresentadas na Prancha 1, deixa claro o padrão habitual sazonal dos atributos climáticos na cidade. Não obstante, também se observa que algumas médias mensais, principalmente, as médias das temperaturas máximas do ar (Gráfico D da Prancha 1), apresentam comportamento fora do habitual, a exemplo das temperaturas registradas no ano de 2010, o único ano da série a registrar um período com temperaturas acima dos $32,0^{\circ} \mathrm{C}$, como registrado no mês de março de 2010 com média de $32,5^{\circ} \mathrm{C}$. 
Os dados mensais de insolação, dias com precipitação e umidade relativa do ar (Gráficos A, B e C da Prancha 1) mostram variabilidade dentro dos padrões habituais, isto é, com ocorrência de totais de dias com precipitação mais elevados e de médias de umidade relativa do ar durante os meses mais chuvosos na cidade, ou seja, entre abril e julho; já as médias mais elevadas de insolação ocorrem nos meses da estação seca: de setembro a janeiro.

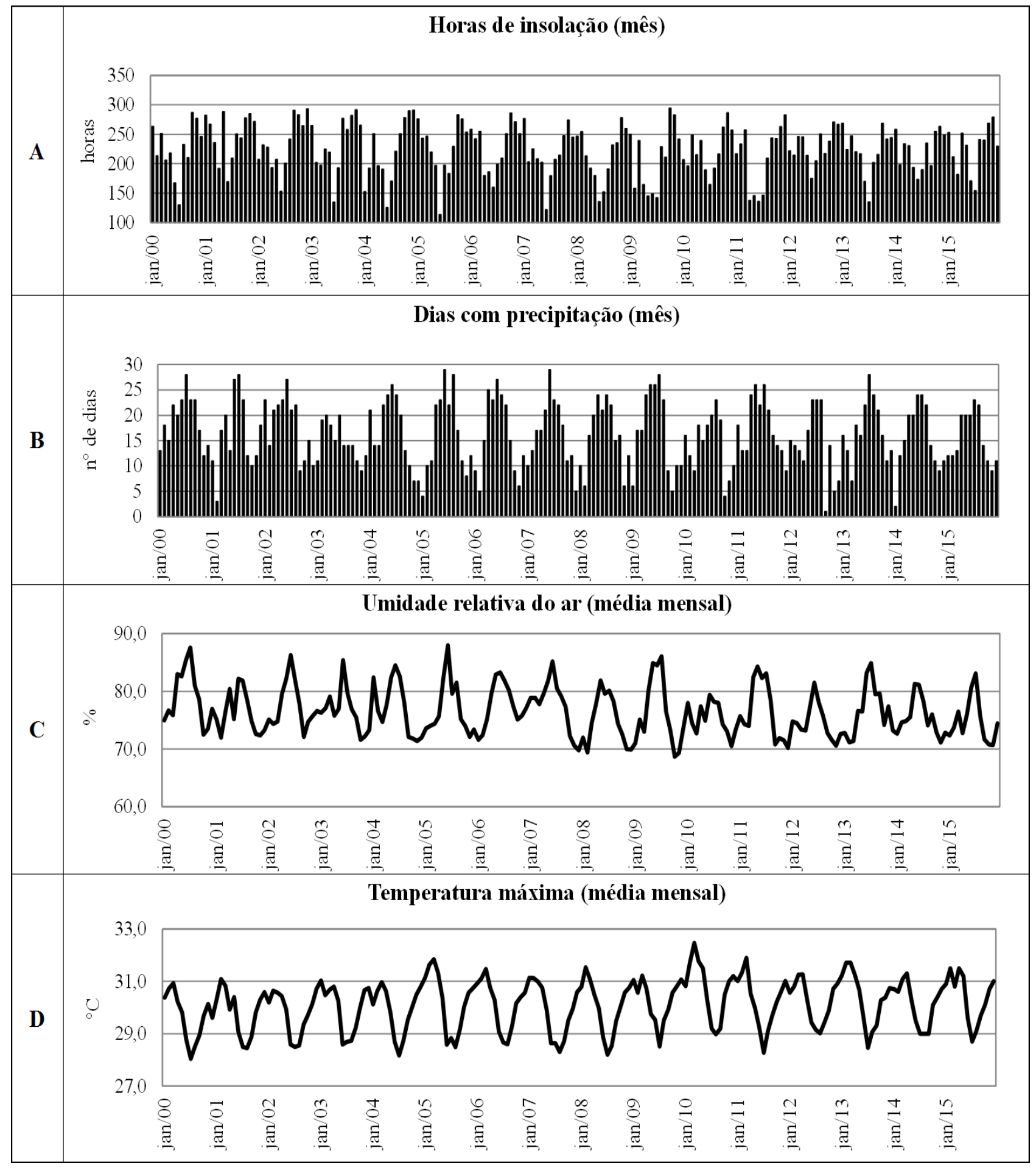

Prancha 1 - Distribuição mensal dos atributos climáticos na cidade de João Pessoa (2000 - 2015). Fonte: BDMEP/ INMET, 2016. Elaboração: Gabriel de Paiva Cavalcante. 


\section{2 - Perfil da morbidade hospitalar por DCV}

No intervalo entre 2000 e 2015, foram registradas 663.889 internações de pacientes residentes na cidade de João Pessoa (DATASUS, 2016). A tabela 1 mostra a taxa média da morbidade hospitalar, por capítulos de doenças da CID -10 e por sexo, dos pacientes residentes em João Pessoa no período de 2000 a 2015, além de também informar o ranking das taxas médias das doenças entre homens e mulheres.

Tabela 1 - Taxa média da hospitalização em João Pessoa, 2000 a 2015.

\begin{tabular}{lcccc}
\hline \multicolumn{1}{c}{ Capítulo CID-10 } & \multicolumn{2}{c}{ Taxa Média } & \multicolumn{2}{c}{ Classificação } \\
& Masculino* & Feminino* & Masculino & Feminino \\
\hline I. Algumas doenças infecciosas e parasitárias & 66,6 & 47,4 & $2^{\circ}$ & $4^{\circ}$ \\
II. Neoplasias (tumores) & 24,1 & 37,8 & $8^{\circ}$ & $7^{\circ}$ \\
III. Doenças do sangue e dos órgãos ematopoéticos e transtornos imunitários & 5,0 & 4,6 & $16^{\circ}$ & $17^{\circ}$ \\
IV. Doenças endócrinas nutricionais e metabólicas & 16,9 & 16,0 & $9^{\circ}$ & $9^{\circ}$ \\
V. Transtornos mentais e comportamentais & 40,3 & 14,7 & $6^{\circ}$ & $11^{\circ}$ \\
VI. Doenças do sistema nervoso & 6,6 & 4,9 & $14^{\circ}$ & $16^{\circ}$ \\
VII. Doenças do olho e anexo & 2,9 & 2,9 & $18^{\circ}$ & $19^{\circ}$ \\
VIII. Doenças do ouvido e da apófise mastóide & 0,4 & 0,4 & $19^{\circ}$ & $20^{\circ}$ \\
IX. Doenças do aparelho circulatório & $\mathbf{5 4 , 3}$ & $\mathbf{5 3 , 2}$ & $4^{\circ}$ & $3^{\circ}$ \\
X. Doenças do aparelho respiratório & 105,6 & 84,8 & $1^{\circ}$ & $2^{\circ}$ \\
XI. Doenças do aparelho digestivo & 52,3 & 43,8 & $5^{\circ}$ & $5^{\circ}$ \\
XII. Doenças da pele e do tecido subcutâneo & 8,6 & 6,1 & $11^{\circ}$ & $15^{\circ}$ \\
XIII. Doenças do sistema osteomuscular e do tecido conjuntivo & 11,4 & 7,8 & $10^{\circ}$ & $12^{\circ}$ \\
XIV. Doenças do aparelho geniturinário & 26,5 & 41,1 & $7^{\circ}$ & $6^{\circ}$ \\
XV. Gravidez, parto e puerpério & 0,0 & 269,5 & $* * *$ & $1^{\circ}$ \\
XVI. Algumas afecções originadas no período perinatal & 6,8 & 6,4 & $13^{\circ}$ & $13^{\circ}$ \\
XVII. Malformações congênitas, deformidades e anomalias cromossômicas & 4,5 & 3,5 & $17^{\circ}$ & $18^{\circ}$ \\
XVIII. Sintomas, sinais e achados anormais (causas mal defínidas) & 7,2 & 6,4 & $12^{\circ}$ & $14^{\circ}$ \\
XIX. Lesões consequentes a causas externas & 66,1 & 23,3 & $3^{\circ}$ & $8^{\circ}$ \\
XX. Causas externas de morbidade e mortalidade & 0,2 & 0,1 & $20^{\circ}$ & $21^{\circ}$ \\
XXI. Contatos com serviços de saúde & 6,2 & 15,1 & $15^{\circ}$ & $10^{\circ}$ \\
\hline
\end{tabular}

*Taxas por 10.000 homens e 10.000 mulheres.

Fonte: DATASUS, 2016. Elaboração: Gabriel de Paiva Cavalcante.

De modo geral, é notável a ocorrência de taxas superiores para as pessoas do sexo masculino: dos 21 grupos de doenças, em 15 a taxa é maior para os homens. Entre os homens, a causa mais comum de morbidade hospitalar é por Doenças do Aparelho Respiratório (105,6 internações por 10 mil homens), conforme mostra a Tabela 1, já entre as mulheres, as internações ocorrem com mais intensidade em decorrência de Gravidez, Parto e Puerpério (269,5 internações por 10 mil mulheres). As Doenças do Aparelho Circulatório, onde se inserem as Doenças Isquêmicas do Coração, compõe a quarta causa de morbidade para as pessoas do sexo masculino e a terceira para as pessoas do sexo feminino, com taxas de 54,3 por 10 mil homens e 53,2 por 10 mil mulheres.

As Doenças Isquêmicas do Coração foram responsáveis por 6.560 internações entre os idosos. Entre as DCV, os dois grupos de Doenças Isquêmicas do Coração (Infarto Agudo do Miocárdio e Outras Doenças Isquêmicas do Coração), foram, de forma respectiva, a terceira e a quarta causa de internações hospitalares. 
Para análise da temporalidade de ocorrência das internações por Doenças Isquêmicas do Coração, a prancha 2 mostra a distribuição mensal e sazonal das taxas médias de internações hospitalares por Doenças Isquêmicas do Coração, segundo sexo dos pacientes idosos e residentes em João Pessoa no período de 2000 a 2015.

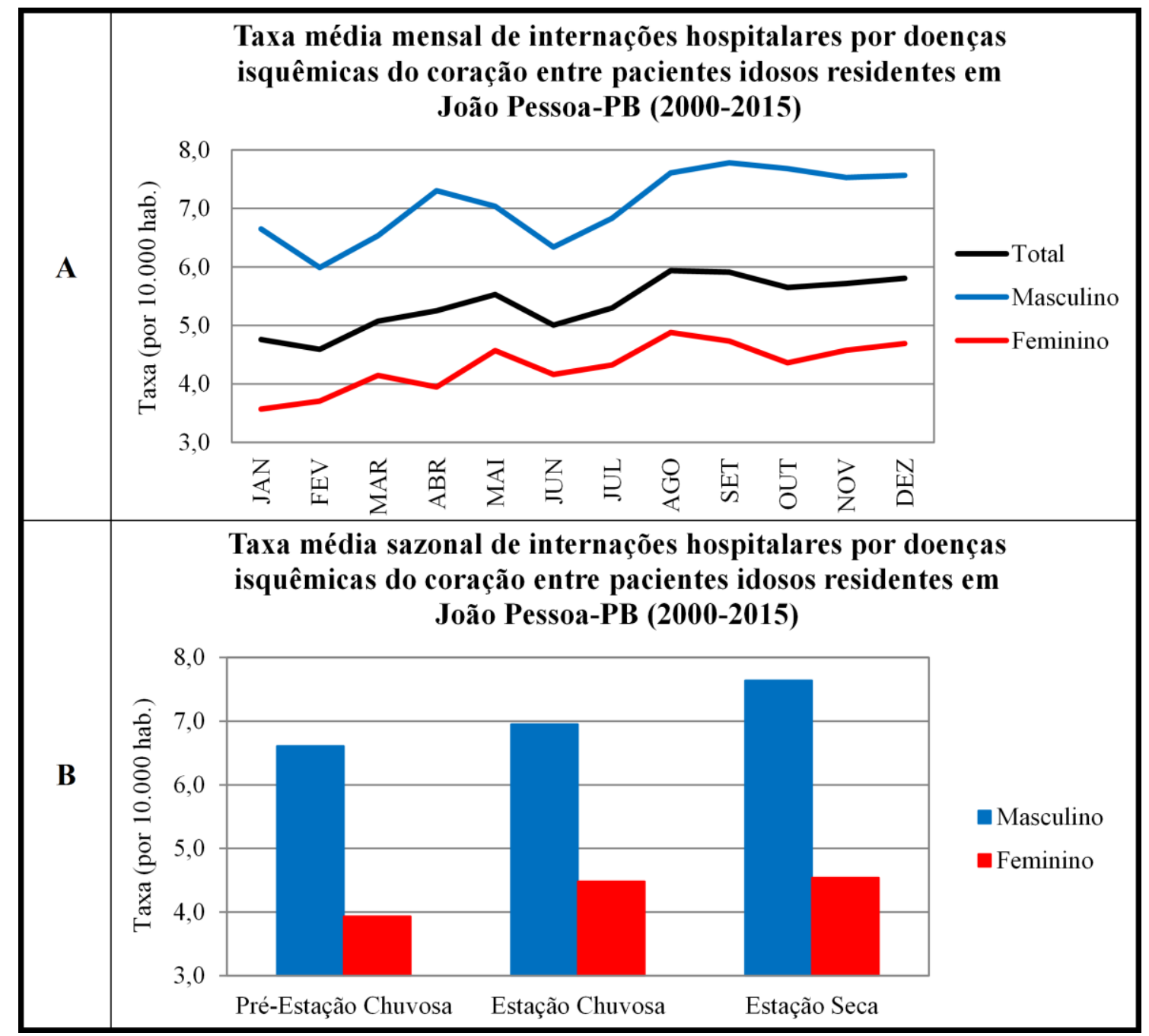

Prancha 2 - Taxas médias das internações mensais e sazonais por Doenças Isquêmicas do Coração entre pacientes idosos em João Pessoa (2000 a 2015).

Fonte: DATASUS, 2016. Elaboração: Gabriel de Paiva Cavalcante.

O gráfico A da prancha 2 mostra um crescimento das taxas médias mensais entre os meses de agosto e dezembro, meses habitualmente mais secos e quentes. Tal crescimento também é visível no comportamento sazonal das taxas médias de internações (Gráfico B da prancha 2), visto que, o período da estação seca apresenta taxa média superior as taxas médias da pré-estação chuvosa e da estação chuvosa. Quanto à distribuição das taxas médias por sexo, observa-se que há predominante superioridade para os idosos do sexo masculino, inclusive com a menor taxa mensal para os homens sendo superior à maior taxa mensal para as mulheres. Na escala sazonal, a superioridade é ainda mais visível. 


\section{3 - Correlações climatopatológicas}

A tabela 2 mostra a correlação mensal entre os atributos climáticos (temperatura máxima, insolação, dias com precipitação e umidade relativa do ar), variáveis explicativas, e as taxas de internações hospitalares por Doenças Isquêmicas do Coração entre pacientes idosos, variável resposta.

Tabela 2 - Correlação de Pearson entre os atributos climáticos e as taxas de internações por Doenças Isquêmicas do Coração entre pacientes idosos residentes em João Pessoa-PB (2000-2015).

\begin{tabular}{lccc}
\hline \multirow{2}{*}{ Atributos Climáticos } & \multicolumn{3}{c}{ Doenças isquêmicas } \\
& Geral & Masc & Fem \\
\hline Temperatura Máxima & $-0,025$ & 0,035 & $-0,088$ \\
Insolação & 0,065 & 0,062 & 0,048 \\
Dias com precipitação & $-0,037$ & $-0,065$ & 0,008 \\
Umidade Relativa do Ar & $-0,091$ & $-0,137$ & $-0,007$ \\
\hline
\end{tabular}

Fontes: DATASUS, 2016 e INMET, 2016. Elaboração: Gabriel de Paiva Cavalcante.

Os valores das correlações entre as variáveis climáticas e as taxas de internações para a população de pacientes idosos foram muito baixos, sendo o coeficiente mais baixo registrado de 0,137 (na correlação entre os dados de umidade relativa do ar e as taxas de internação para idosos do sexo masculino), e o mais alto, de 0,065 (para a correlação entre os dados mensais de insolação e as taxas de internação sem distinção de sexo). Apenas o atributo climático Insolação registrou todos os coeficientes positivos, porém muito próximos de 0 , ou seja, correlação praticamente nula. A título de exemplo podemos visualizar no gráfico 1 o baixo grau de relação entre temperatura máxima do ar e as taxas de internações hospitalares sem distinção de sexo. As taxas de internações por Doenças Isquêmicas do Coração dos pacientes idosos não sinalizam relação com as temperaturas máximas mensais registradas, resultando em uma relação do tipo "nuvem”, ou seja, em termos estatísticos, uma situação na qual não ocorre ralação direta entre os dados.

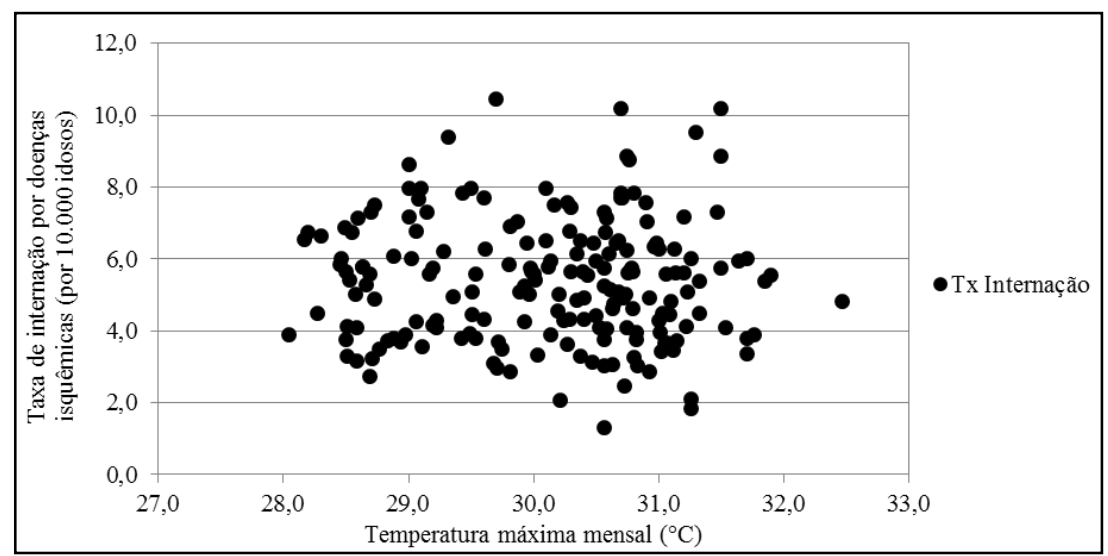

Gráfico 1 - Taxa mensal de internações hospitalares por Doenças Isquêmicas do Coração entre pacientes idosos versus a temperatura média mensal do ar em João Pessoa (2000-2015).

Fontes: DATASUS, 2016 e INMET, 2016. Elaboração: Gabriel de Paiva Cavalcante. 
As próximas análises utilizarão os dados de temperatura máxima para as correlações com os dados de internações hospitalares. Na escala sazonal foram identificadas correlações baixas entre as variáveis, conforme mostra a tabela 3. Os coeficientes de correlação não apresentaram significância estatística de acordo com o Teste t de Student. As correlações variaram entre -0,11 (coeficiente para o sexo feminino na Estação Seca) e 0,238 (coeficiente para o sexo masculino na Pré-Estação Chuvosa). Mesmo sem a significância estatística, é notável que a Pré-Estação Chuvosa apresenta os maiores coeficientes dos regimes sazonais. Por outro lado, os registros de correlação da Estação Seca são negativos.

Tabela 3 - Correlação de Pearson entre a temperatura máxima do ar e as taxas de internações por Doenças Isquêmicas do Coração entre pacientes idosos residentes em João Pessoa-PB na escala sazonal (2000-2015).

\begin{tabular}{|c|c|c|c|}
\hline Doenças & & Estação & \\
\hline Isquêmicas & Pré-Estação Chuvosa & Estação Chuvosa & Estação Seca \\
\hline Geral & 0,224 & 0,087 & $-0,053$ \\
\hline Masculino & 0,238 & 0,113 & $-0,080$ \\
\hline Feminino & 0,215 & 0,135 & $-0,110$ \\
\hline
\end{tabular}

Quanto às correlações mensais, presentes na tabela 4, registram-se coeficientes de correlação de grau baixo a moderado, porém, sem significância estatística. Os coeficientes de correlação variaram entre $-0,23$ (coeficiente para o sexo feminino no mês de agosto) e 0,45 (coeficiente para o sexo masculino no mês de abril e para a taxa de morbidade geral no mês de maio).

Tabela 4 - Correlação de Pearson entre a temperatura máxima do ar e as taxas de internações por Doenças Isquêmicas do Coração entre pacientes idosos residentes em João Pessoa-PB na escala mensal (2000-2015).

\begin{tabular}{cccccccccccccc}
\hline Doenças & \multicolumn{11}{c}{ Mês } \\
Isquêmicas & JAN & FEV & MAR & ABR & MAI & JUN & JUL & AGO & SET & OUT & NOV & DEZ \\
\hline Geral & 0,04 & 0,29 & 0,13 & 0,33 & 0,45 & 0,17 & 0,11 & $-0,14$ & 0,19 & 0,42 & $-0,01$ & 0,02 \\
Masculino & 0,02 & 0,33 & 0,12 & 0,45 & 0,44 & 0,22 & 0,21 & $-0,01$ & 0,20 & 0,41 & $-0,21$ & 0,14 \\
Feminino & 0,07 & 0,09 & 0,11 & 0,16 & 0,31 & 0,04 & $-0,08$ & $-0,23$ & 0,13 & 0,35 & 0,20 & $-0,14$ \\
\hline \multicolumn{7}{l}{ Fontes: DATASUS, 2016 e INMET, 2016. Elaboração: Gabriel de Paiva Cavalcante. }
\end{tabular}

Para cada ano da série, os dados de correlação (Tabela 5) registraram variações tanto para correlações diretamente proporcionais, quanto para correlações inversas. Cabe destacar que nenhum coeficiente de correlação apresentou significância estatística de acordo com o teste t de Student. Para as correlações diretas, destaca-se o ano de 2003, sobretudo, para as taxas hospitalares da população total idosa e para as taxas do sexo masculino, com coeficientes de 0,49 e 0,58, respectivamente. O ano de 2007 mostrou características semelhantes, porém, com grau de associação menor. Já nas correlações inversas, destacam-se os anos de 2012 e 2013, com coeficientes entre $-0,3$ e $-0,65$. As correlações registradas para a população idosa do sexo feminino 
foram inversas e/ou próximas a 0 , não relevando grau de associação relevante no que diz respeito à estatística. O maior registro de correlação positiva ocorreu no ano de $2002(0,29)$, enquanto que a correlação negativa mais relevante ocorreu no ano de $2004(-0,64)$.

Tabela 5 - Correlação de Pearson entre a temperatura máxima do ar e as taxas de internações por Doenças Isquêmicas do Coração entre pacientes idosos residentes em João Pessoa-PB na escala anual (2000-2015).

\begin{tabular}{|c|c|c|c|c|c|c|c|c|c|c|c|c|c|c|c|c|}
\hline \multirow{2}{*}{$\begin{array}{c}\text { Doenças } \\
\text { Isquêmicas }\end{array}$} & \multicolumn{16}{|c|}{ Ano } \\
\hline & 2000 & 2001 & 2002 & 2003 & 2004 & 2005 & 2006 & 2007 & 2008 & 2009 & 2010 & 2011 & 2012 & 2013 & 2014 & 2015 \\
\hline Geral & $-0,53$ & $-0,56$ & 0,11 & 0,49 & $-0,53$ & $-0,01$ & $-0,12$ & 0,33 & $-0,17$ & $-0,08$ & $-0,02$ & 0,30 & $-0,52$ & $-0,42$ & $-0,11$ & 0,08 \\
\hline Masculino & $-0,10$ & $-0,63$ & $-0,06$ & 0,58 & 0,04 & $-0,07$ & $-0,29$ & 0,49 & $-0,08$ & 0,37 & $-0,08$ & 0,18 & $-0,65$ & $-0,53$ & $-0,01$ & 0,24 \\
\hline Feminino & $-0,60$ & $-0,18$ & 0,29 & 0,20 & $-0,64$ & 0,05 & 0,08 & $-0,04$ & $-0,12$ & $-0,53$ & 0,09 & 0,18 & $-0,30$ & $-0,33$ & $-0,19$ & $-0,17$ \\
\hline
\end{tabular}

Utilizando uma abordagem de síntese (média anual dos registros de temperatura máxima e taxa média anual de internações por Doenças Isquêmicas do Coração) entre todos os anos da série (2000 a 2015), encontram-se os maiores registros de correlação, o que leva a inferir que, embora a análise dos dados seja a mais generalizada do trabalho, os anos mais quentes registram, também, as maiores taxas de internações hospitalares, sobretudo para os idosos do sexo masculino (Tabela 6), que registrou correlação de 0,375 . Para as taxas gerais de internação, o coeficiente foi de 0,303 , e para o sexo feminino, 0,155. Vale destacar que, embora sejam os dados de correlação mais relevantes, o teste $\mathrm{t}$ de Student também não apontou significância estatística.

Tabela 6 - Correlação de Pearson entre a temperatura máxima do ar e as taxas de internações por Doenças Isquêmicas do Coração entre pacientes idosos residentes em João Pessoa-PB na síntese anual (2000-2015).

\begin{tabular}{|c|c|c|c|}
\hline \multirow{2}{*}{ Atributo Climático } & \multicolumn{3}{|c|}{ Doenças isquêmicas } \\
\hline & Geral & Masc & Fem \\
\hline Temperatura Máxima & 0,303 & 0,375 & 0,155 \\
\hline
\end{tabular}

Fontes: DATASUS, 2016 e INMET, 2016. Elaboração: Gabriel de Paiva Cavalcante.

\section{CONSIDERAÇÕES FINAIS}

As correlações do período de 2000 a 2015 entre as médias dos atributos climáticos, variáveis explicativas, e as taxas médias de internações hospitalares por Doenças Isquêmicas do Coração entre pacientes idosos, variável resposta ou variável desfecho da análise mostraram um grau de associação muito baixo e sem significância estatística. Este mesmo padrão foi identificado nas correlações sazonais entre a temperatura máxima do ar e taxas hospitalares. Quanto às correlações mensais e anuais entre a temperatura máxima do ar e taxas hospitalares verificou-se grau de associação de baixo a moderado, mas sem significância estatística conforme apontou o teste t de Student. Já para a escala de síntese anual (média anual dos registros de temperatura máxima e taxa média anual de internações por Doenças Isquêmicas do Coração) ocorrem os maiores coeficientes, 
o que nos permite afirmar que os anos mais quentes registram, também, as maiores taxas de internações hospitalares por doenças isquêmicas do coração.

Nesse sentido, podemos afirmar, com base nos recursos estatísticos utilizados neste trabalho, que as médias mensais de temperatura máxima do ar, de horas de insolação, de dias com precipitação e médias de umidade relativa do ar não produziram um impulso de natureza ambiental no aumento ou na diminuição da morbidade hospitalar por Doenças Isquêmicas do Coração entre pacientes idosos, atendidos em hospitais públicos e conveniados ao SUS e residentes na cidade de João Pessoa-PB. Por outro lado, os anos mais quentes também registraram as maiores taxas de internações, o que pode comprovar a associação direta entre a diminuição do conforto térmico na cidade e o aumento das taxas médias anuais de internações.

\section{REFERÊNCIAS}

\section{BDMEP/INMET. Banco de Dados Meteorológicos para Ensino e Pesquisa/Instituto Nacional de Meteorologia. Disponível em:} http://www.inmet.gov.br/portal/index.php?r=bdmep/bdmephttp://www.inmet.gov.br/portal/index.ph p?r=bdmep/bdmep. Acesso em: 06 ago. 2014.

BESANCENOT, J. P. Infarctus du myocarde, saisons et climats. In: Revue de Géographie de Lyon. vol. 61, $\mathrm{n}^{\circ} \quad 3$, p. $271 \quad$ - 281, 1986. Disponível em: http://www.persee.fr/web/revues/home/prescript/article/geoca_0035113X_1986_num_6134097. Acesso em: 15 jul. 2012.

BESANCENOT, J. P. Climat et santé (Coll. "Médecine et Société). Paris: PUF, 2001. 128 p.

BESANCENOT, J. P. Vagues de chaleur et mortalité dans les grandes agglomérations urbaines. Environnement, Risques \& Santé, v 1, n. 4, p. 229-40, Sep./ Oct. 2002.

DATASUS. Departamento de Informática do SUS. Disponível em: http://www.datasus.gov.br/DATASUS/index.php. Acesso em: 01 ago. 2015.

FIGUEIREDO FILHO, D. B.; SILVA JÚNIOR, J. A. Desvendando os mistérios da correlação de Pearson (r). Revista Política Hoje, vol. 18, n. 1, p. 115-146, 2009.

GALVÃO, N; LEITE, M. L. VIRGENS FILHO, J. S.; PONTES, C. C. Relação entre fatores climáticos e doenças do aparelho cardiovascular no município de Ponta Grossa-PR. Revista Hygeia (online),11 (21),p. 93 - 106, 2015.

IBGE. Instituto Brasileiros de Geografia e Estatística. Disponível em: <http:// www.ibge.gov.br>. Acesso em: 01 jul. 2015.

LIMA, J. R. C; PORDEUS, A. M. J; ROUQUAYROL. M. Z. Medida em saúde pública, p.25 - 64. In: ROUQUAYROL. M. Z; GURGEL, M. (Orgs.) Epidemiologia \& Saúde. 7 ed. Rio de Janeiro: MedBook, 2013. 736p. 
MC MICHAEL, A. J. et al. International study of temperature, heat and urban mortality: the ISOTHURM $^{\text {ee }}$ Project. International Journal of Epidemiology, n. 37, p.1121-1131. 2008 Disponível em: <http:// www.heart.bmj.com>. Acesso em: 12 set. 2014.

MOURA, M. O. Climatopatologia por meteorossensibilidade em Fortaleza, Ceará, p.67 -96. In: ZANELLA, M. E; SALES, M.C. L (Orgs.). Clima e recursos hídricos no Ceará na perspectiva geográfica. Fortaleza: Expressão Gráfica e Editora, 2015. 204p.

MOURA, M. O. Anomalias das Temperaturas Extremas do Ar em Fortaleza: correlações com a morbidade hospitalar por doenças cardiovasculares. (Tese de Doutorado). Fortaleza: Programa de Pós-Graduação em Geografia/UFC, 2013. 248p.

MURARA, P; COELHO, M. S. Z. S.; AMORIM, M. C. C. T. Análise da influência meteorológica nas internações por doenças cardiovasculares. Caderno Prudentino de Geografia, v. 1, p. 55-65, 2010.

SANT'ANNA NETO, J. L. A. As Matrizes da Construção da Climatologia Geográfica Brasileira, p. 7-60. In: MONTEIRO, C. A. F.; MENDONÇA, F. A.; ZAVATTINI, J. A.; SANT'ANNA NETO, J. L. A. (Orgs.) Construção da Climatologia Geográfica no Brasil. Campinas: Alínea, 2015, $194 \mathrm{p}$.

PAIXÃO, E. J; NOGUEIRA, P. J. Efeitos de uma onda de calor na mortalidade. Revista Portuguesa de Saúde Pública, v.21, n.1, 2003, p. 41-54. Disponível em: <http://www.ensp.unl.pt/publicacoes>. Acesso em: 21 set. 2014.

PEREIRA, H.S. et al. Correlação de variáveis meteorológicas com o infarto agudo do miocárdio. In: IV Simpósio Internacional de Climatologia. João Pessoa/PB, 2011. CD ROM.

PITTON, S. E. C; DOMINGOS, A. E. Tempo e doenças: efeitos dos parâmetros climáticos nas crises hipertensivas nos moradores de Santa Gertrudes- SP. Estudos Geográficos, Rio Claro, n. 2, p. 75-86, jun. 2004. Disponível em: <http://www.rc.unesp.br/igce/grad/geografia/revista.htm>. Acesso em: 19 nov.2015.

TRIOLA, M. F. Introdução à estatística. 10 ed. Rio de Janeiro: Livros Técnicos e Científicos Editora S.A., 2008. 696p.

WHO. Health and global environmental change (Heat-waves: risks and responses). World Health Organization, 2004. 124p. Disponível em: 〈http:// www.euro.who.int/globalchange>. Acesso em: 23 ago. 2014.

Recebido em: 14/08/2016

Aceito para publicação em: 01/10/2016 